\title{
Prediction of the osmotic/activity coefficients of alkali hydroxide electrolytes
}

\author{
Timothy T. Duignan* and X. S. Zhao* \\ School of Chemical Engineering, The University of Queensland, St Lucia, Brisbane 4072, \\ Australia \\ E-mail: t.duignan@uq.edu.au; george.zhao@uq.edu.au
}

\begin{abstract}
The osmotic/activity coefficients are one of the most fundamental and important properties of electrolyte solutions. There is currently no reliable means of predicting them from first principles without relying on extensive fitting to experimental data. The alkali hydroxide aqueous electrolytes are a particularly important class of solutions due to the crucial role they play in a vast range of applications. Here, for the first time we predict the osmotic/activity coefficients of these solutions without any fitting using a previously developed continuum solvent model of ion-ion interactions without modifications. The feasibility of making these predictions with first principles molecular simulation is also assessed. This work demonstrates the reliability of the continuum solvent model and provides a plausible pathway to the fast and accurate prediction of these important quantities for a wide range of electrolyte solutions. It also implies that an explicit description of the structure of water molecules around ions may not be required to predict important thermodynamic properties.
\end{abstract}

Keywords: Chemical equilibria, continuum solvent model, electrolyte solutions. 


\section{Introduction}

Aqueous alkali hydroxide solutions play a central role in a huge number of important applications. For example, aqueous alkali hydroxides are a standard electrolyte used in supercapacitors due to their high conductivity ${ }^{1}$ as well as the fact that they can enable pseudocapacitance. ${ }^{2,3}$ The hydroxide ion is also thought to play a key role in water in salt electrolytes where it can facilitate the formation of a beneficial solid electrolyte interphase. ${ }^{4}$ Additionally, hydroxide anions are involved in the chemical equilibria of a vast range of systems. For instance, aqueous alkali hydroxides are a key component in a promising process for capturing $\mathrm{CO}_{2}$ from air, where the use of potassium hydroxide instead of sodium hydroxide leads to a significant performance improvement. ${ }^{5}$ Similarly, the reactivity of hydroxide ions in bulk and at the electrode interface is key to the electrocatalytic reduction of $\mathrm{CO}_{2} \cdot{ }^{6-8}$

A fundamentally important property of these electrolytes is their osmotic/activity coefficients. The activity coefficients can be directly related to the osmotic coefficients through the Gibbs-Duhem Relationship. These coefficients determine a host of important properties. As the activity coefficients are key to determining the chemical potential they are also key to predicting chemical equilibria and solubilities. ${ }^{9}$ They are also an important measure of ion pairing, ${ }^{10,11}$ which is known to be important for determining conductivities and many Hofmeister effects. ${ }^{9,12}$ They are also crucial in determining reaction rates through the Bjerrum-Brønsted equation. ${ }^{13}$

Given their importance, it is remarkable how little is known about the osmotic/activity coefficients of these solutions. For example, we are not aware of any attempts to predict these activity coefficients theoretically without relying on experimental fitting to the activity coefficients themselves. In fact using first principles methods to predict osmotic/activity coefficients has seen little progress in many years. This means that in order to build thermodynamic models of the huge range of practical systems where these equations are important, systems of equations with parameters fitted to experiment have to be used. Gaps and reliability problems with this experimental data mean that this approach is very problematic. ${ }^{14,15}$ 
Additionally, many of the experimental measurements of these quantities were performed over a half century ago and, to the best of our knowledge, have not been revisited since then. ${ }^{16}$ The reliability of these measurements is unclear considering the difficulty of carrying them out and the underlying assumptions the measurements rely on. Additionally, rubidium hydroxide osmotic/activity coefficients appear to have never been measured.

Careful simulation of hydroxide ions in water using sophisticated quantum Density Functional Theory based Molecular Dynamics (DFT-MD) have been performed. ${ }^{17-19}$ These simulations provide a wealth of information about the atomistic details of these ions in water such as the orientations and structures that the surrounding water molecules occupy. But these methods have not been used to determine thermodynamic properties such as osmotic/activity coefficients.

Here, we apply a relatively simple continuum solvent model (CSM), which was previously applied to the alkali halide ions in water, to the alkali hydroxide salts in water. ${ }^{11,20,21}$ This model can be directly applied with no modification and no new parameters. It is able to reproduce the osmotic/activity coefficients of these electrolytes well. We also perform DFT-MD simulations of the sodium hydroxide salt. These results show that there is a significant dependence on which DFT functional is used. Additionally, the CSM gives better experimental agreement for the osmotic/activity coefficients. This is significant as it indicates that a simple continuum solvent models can be used to estimate properties that cannot or have not been adequately determined experimentally quickly and easily. It also implies that a detailed understanding of the atomistic structure of water molecules around an ion may not be required to predict important thermodynamic properties with good accuracy. A comparison of the behaviour of $\mathrm{OH}^{-}$with fluoride is also provided. 


\section{Theory}

\section{Continuum solvent model}

Methodological details for the CSM are provided in full in previous publications. ${ }^{11,20,21}$ In brief, the ion-ion interaction free energies are computed from the following expression:

$$
W(r)=\frac{1}{\lambda}\left(W_{\mathrm{COSMO}}(r)+\Delta W_{\mathrm{cav}}(r)+\Delta W_{\mathrm{disp}}(r)+\Delta W_{\mathrm{CT}}(r)\right)
$$

where $W_{\text {COSMO }}(r)$ corresponds to the quantum mechanical ion-ion interaction calculated with MP2 level of theory of the two ions combined with an approximate treatment of the electrostatic effect of the surrounding continuum modelled using the conductor like screening model (COSMO). $\Delta W_{\text {cav }}(r)$ is a surface area dependent term aimed at approximately accounting for cavity formation energy effects. $\Delta W_{\text {disp }}(r)$ captures the loss of ion-water dispersion interaction as the ions come into contact and $\Delta W_{\mathrm{CT}}(r)$ is also a surface area dependant term to correct for the neglect of charge transfer between the ion and the surrounding solvent with the continuum solvent treatment. This whole interaction is also damped by a factor $\lambda$ which aims to correct for the over-attraction caused by the charge transfer between the two ions.

This $W_{\text {COSmo }}$ term depends on the orientation of the hydroxide ion relative to the alkali cation. All of these terms are computed using previously published parameters and methods. For instance, the cavity size and solvation free energy values for hydroxide are given in Ref 20 and for the cations are given in Ref. 11. Following Ref. ${ }^{20}$ we use an isotropic approximation for all of the terms. This means that only $W_{\mathrm{COSMO}}(r)$ depends on the orientation of the

ions. In order to determine an isotropic version of $W_{\mathrm{COSMO}}(r)$ we thermally average using the following equation: ${ }^{20}$

$$
W_{\mathrm{COSMO}}(r)=-\frac{k_{\mathrm{B}} T}{2} \ln \left(\int_{0}^{\pi} d \theta \sin \theta \exp ^{-\beta W_{\mathrm{COSMO}}(r, \theta)}\right)
$$


We also have to adjust the potential slightly at large separation to match the Coulomb attraction. We treat the like charge interactions with a simple Coulomb repulsion and hard sphere repulsion determined by the ions crystal radii. Explicit modelling of like charge interaction is neglected following Ref. 11 due to the fact that short range like ion interactions make a small contribution to activity/coefficients in this low/moderate concentration range. ${ }^{22}$

\section{Results \& Discussion}

The $W_{\mathrm{COSMO}}(r)$ interaction for sodium hydroxide as a function of orientation is shown in Figure 1. It shows a strong preference for the oxygen to orient towards the cation as expected from the dipole moment on the hydroxide.

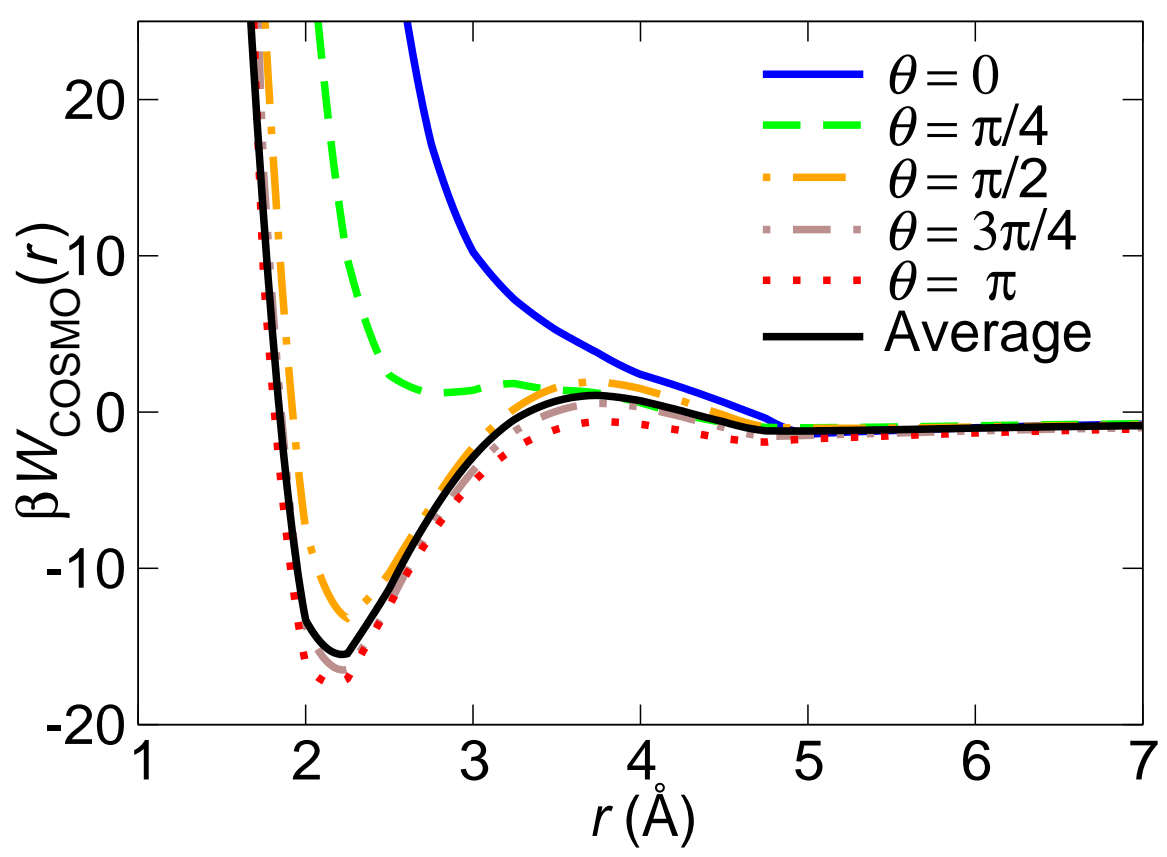

Figure 1: COSMO calculation of the interaction between sodium and hydroxide ions as a function of orientation. $\theta=0$ corresponds to the $\mathrm{OH}$ bond pointing towards sodium. The Boltzmann averaged interaction is also provided. 
The full potentials of mean force (PMFs) computed with the CSM for all the alkali hydroxide electrolytes are shown in Figure 2. They show substantial energy cost to dehydrate the ions and favourable contact ion pair formation for small ion pairs, qualitatively consistent with the behaviour observed for alkali fluorides in water. ${ }^{11}$

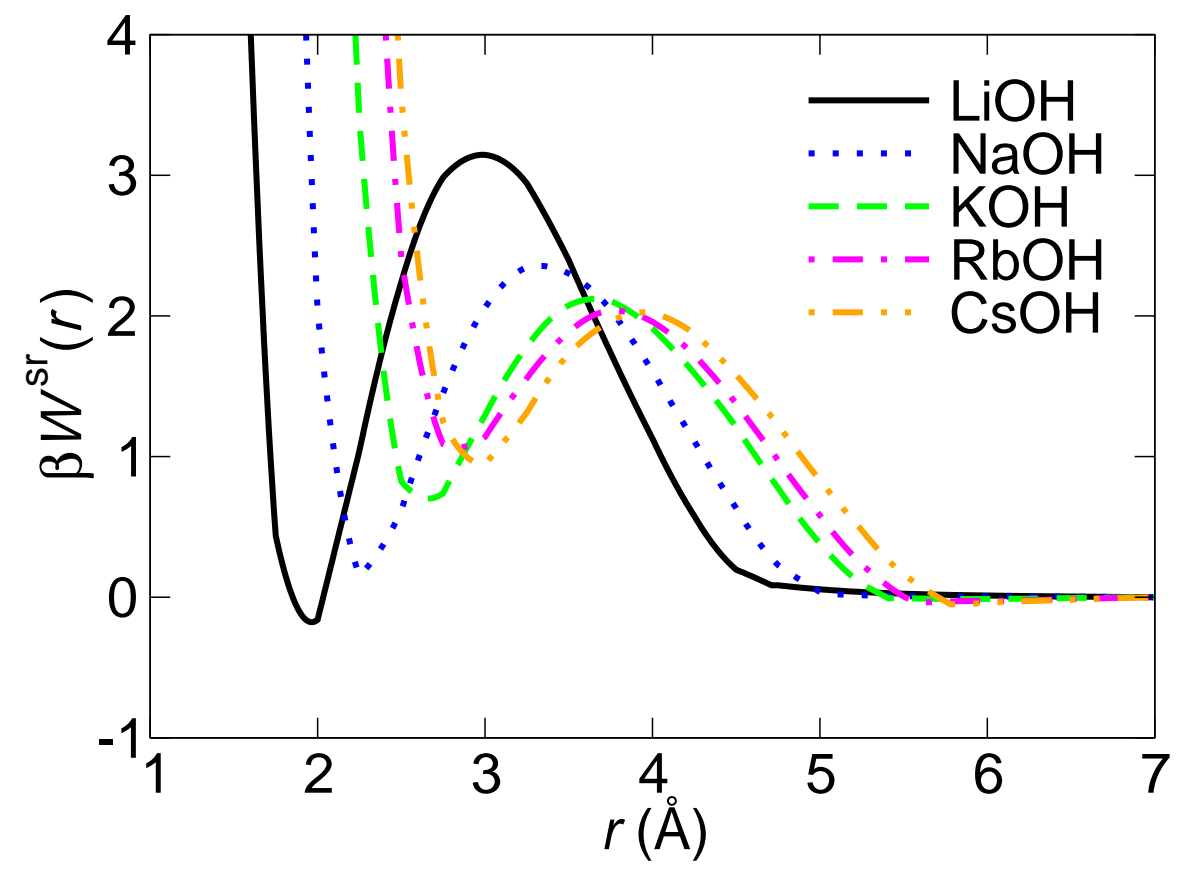

Figure 2: Total short range interaction potentials for alkali hydroxide ion pairs calculated using the CSM. (The long range Coulomb interaction has been subtracted)

This PMF computed with the CSM is an infinite dilution PMF. It can be used to predict osmotic coefficients using the Hyper-netted Chain (HNC) closure of the Ornstein-Zernike (OZ) equation as described in the calculation details given below. The resulting predictions are shown in Figure 3. The comparison with the activity coefficients is essentially the same. Excellent agreement for sodium and potassium hydroxides is observed. This is remarkable considering no fitting has been used to reproduce these and that these coefficients are notoriously sensitive to small differences in the PMF. ${ }^{22}$ Lithium agrees at low concentration cesium is too high at most concentrations in comparison to experiment.

The experimental values used here are determined by the Pitzer equations, which are 


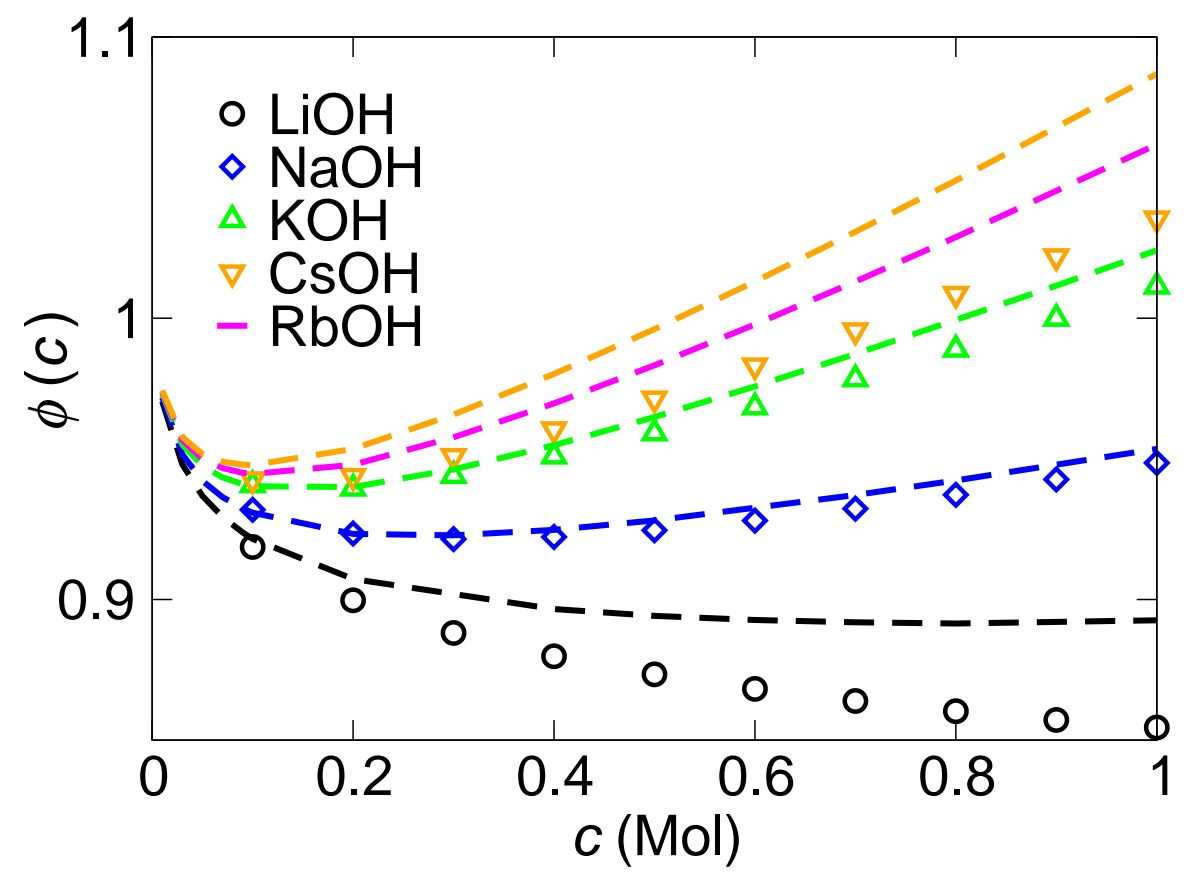

Figure 3: Osmotic coefficients of the alkali hydroxides calculated with the continuum solvent model (dashed lines) compared with experiment as a function of concentration. (Symbols) 
fitted to provide a close match to experimental measurements. ${ }^{16}$ However, as Pitzer points out, it is worth noting that the measurement of these coefficients for hydroxides is challenging and more difficult than for normal salts. While the values for $\mathrm{NaOH}$ are fairly consistent in the literature. The values for lithium and cesium show some variation. This is due to the reliance of amalgam mercury electrodes which can be unreliable or the use of electrolyte mixtures which require modelling to extract these values. For example the value for cesium hydroxide reported by Robinson and Stokes at $0.1 \mathrm{M}$ is actually substantially higher than the theoretical value determined here. ${ }^{9}$ It would also be quite surprising if cesium hydroxide was so similar to potassium given the large difference observed for the similar alkali fluoride electrolytes. Additionally, rubidium appears to have never been measured. The values given here constitute the first predictions of this quantity and demonstrate the utility of a simple computational model of electrolyte solutions. These measurements are also very old at over 50 years new experiments to resolve the discrepancies in this old data and to provide a value for the rubidium hydroxide electrolyte to test the models predictive ability would be very useful.

The plot comparing the values at $0.5 \mathrm{M}$ is prepared in Figure 4 . This shows that the agreement is almost as good for the hydroxide electrolytes as for the alkali halide ions with a small overestimate for cesium and lithium.

Hellström and Behler ${ }^{18}$ have performed molecular dynamics simulation of $\mathrm{NaOH}$ solution using a neural network based force field (NN-MD) trained on DFT calculations. These simulations are consistent with neutron scattering data. The structural details observed in these simulations, such as the dehydration barrier width and height, are not well reproduced with the CSM as previously reported for the case of $\mathrm{NaCl}^{21}$ and as expected due to the significant approximation associated with the CSM.

The NN-MD simulation cannot be used to directly compute activities itself since the PMF at infinite dilution is required and the box size needs to be more than $40 \mathrm{~nm}$ to correctly reproduce the long range Coulomb interaction observed at infinite dilution. ${ }^{22}$ However, we 


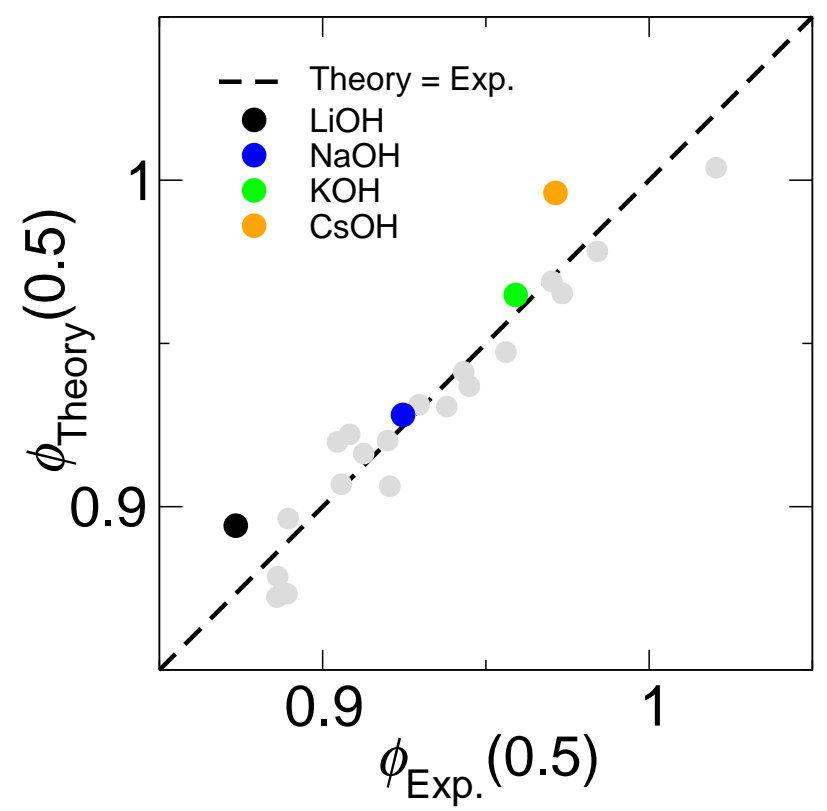

Figure 4: Osmotic coefficients at $0.5 \mathrm{M}$ calculated with the continuum solvent model compared with experiment at $0.5 \mathrm{M}$. Alkali halides are in grey. 
can compute the osmotic coefficients for the NN-MD approach using the protocol developed in Ref. ${ }^{21}$ This approximates the infinite dilution PMF by using the Radial Distribution Function $(\mathrm{RDF})$ at the lowest concentration simulation and by taking its negative natural log to and combining it with a long range Coulomb interaction beyond 7 angstroms. Then using the same OZ solver as used for the CSM PMFs the osmotic coefficients can be calculated. As shown in Figure 5, this protocol gives reasonable agreement with experimentally measured osmotic coefficients although, as for the $\mathrm{NaCl}$ case, they becomes somewhat too large at higher concentrations. ${ }^{21}$ Remarkably the CSM gives better agreement even than this much more computationally expensive approach.

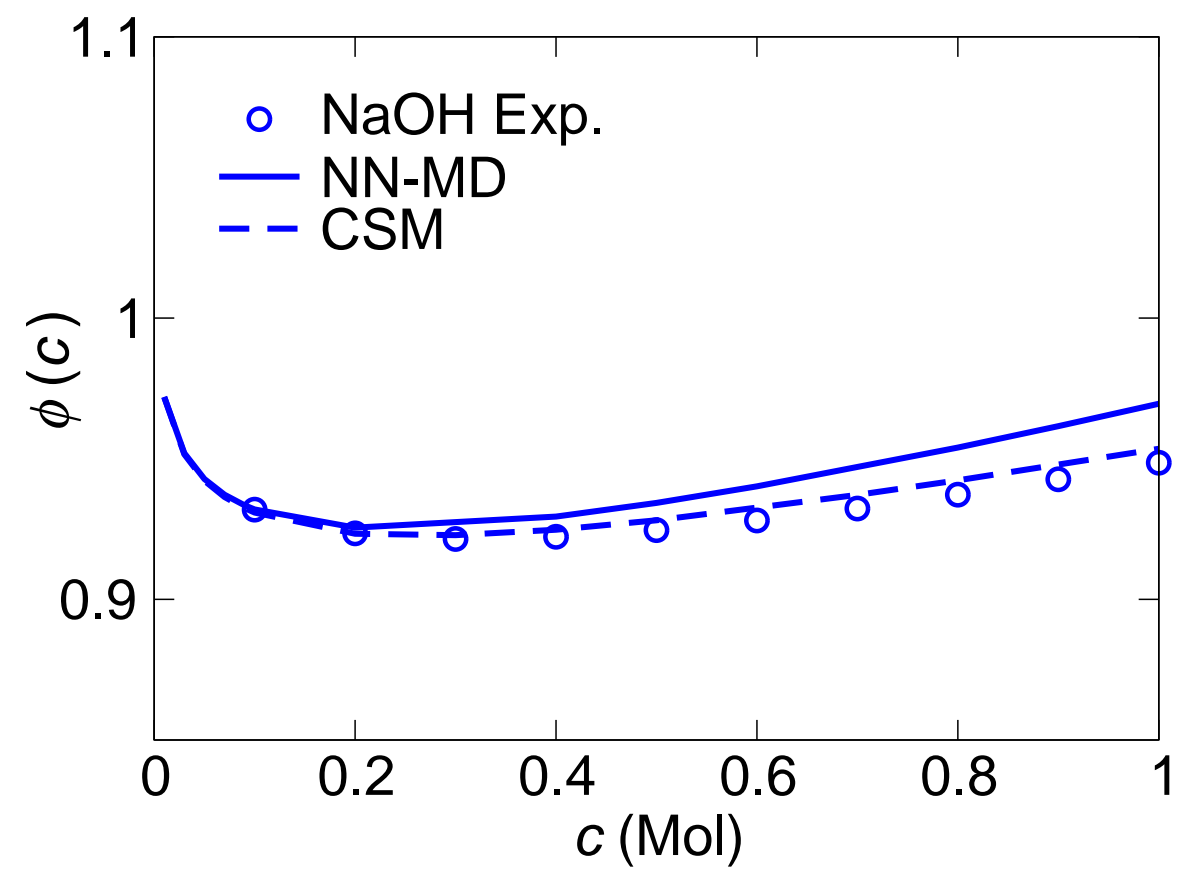

Figure 5: Osmotic coefficients calculated with the neural net DFT method and the protocol outlined previously in ${ }^{11}$ compared with experiment and the CSM.

A challenge with using first-principles based simulations to determine this quantity that there can be a substantial variation based on which DFT functional is used to run the simulations. For example, Figure 6 shows the RDF computed using the revPBE-D3 functional to simulate a sodium hydroxide solution compared with the RDF of the NN-MD potential. ${ }^{18}$ 
It is clear that there are substantial differences. These can be attributed to the fact that it is know that uncorrected revPBE-D3 is too repulsive on the first hydration shell around the sodium cation. ${ }^{23,24}$ This results in the water molecules residing further away from the sodium. This explains why the peaks in the $\mathrm{NaOH}$ PMF are shifted by a similar amount and why too much contact pair formation is observed relative to the NN-MD calculation. Remarkalby, the NN-MD force field is trained using the RPBE-D3 DFT functional, which should be very similar to revPBE-D3. It is therefore necessary to develop more sophisticated methods of simulating electrolyte solutions with DFT-MD. For example, by using higher level functionals or by including correction potentials adjusted to minimise the error in higher level cluster calculations. ${ }^{24,25}$

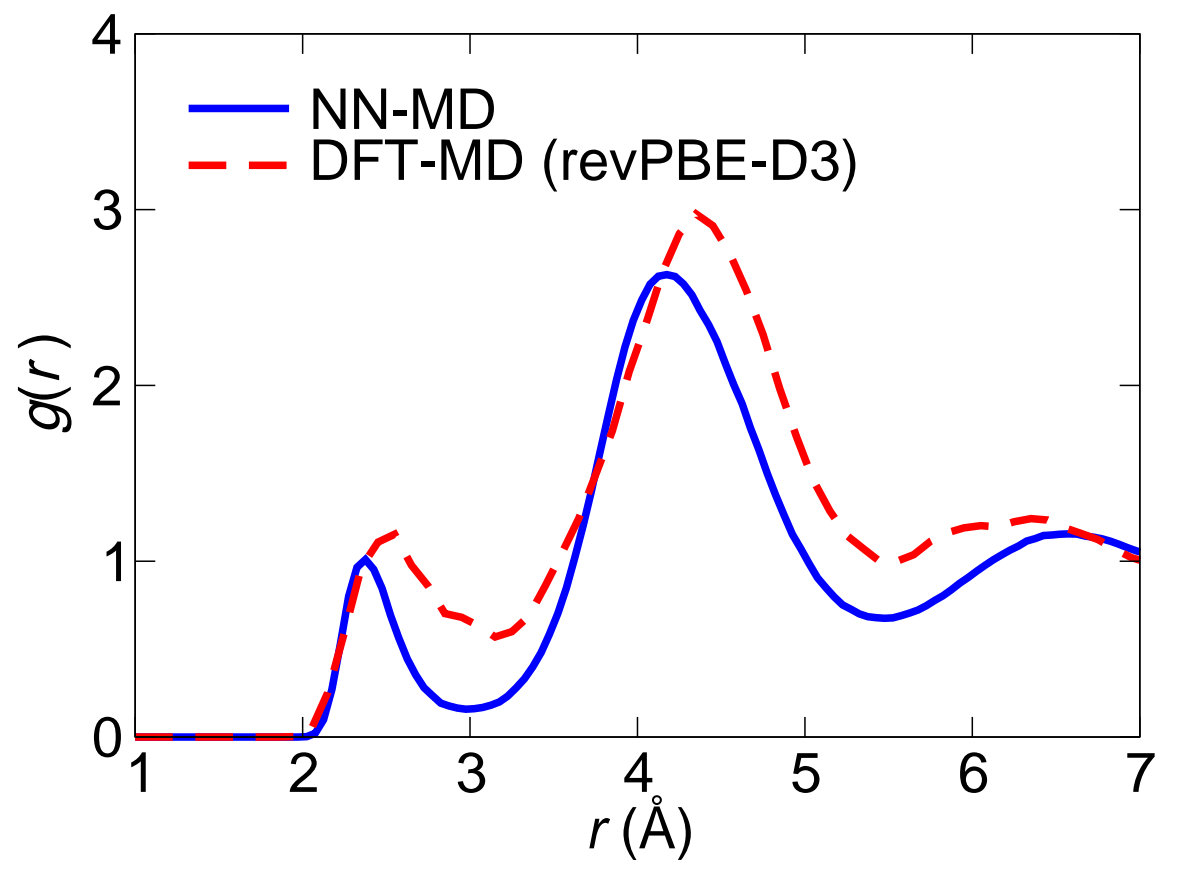

Figure 6: Comparison of the Na-Cl RDF determined with two different first principles based simulation methods. The NN-MD RDF is taken from Ref. 18 at 2.60 M. The DFT-MD is at 2.3 M. Calculation details for DFT-MD are given below.

Finally, Figure 7 shows the characteristic V-shape plot of the osmotic/activity coefficients of the alkali halide series which is indicative of the so called 'law' of matching water affini- 
ties. ${ }^{26}$ This 'law' states that ions of similar hydration free energy have the strongest ion-pair formation propensity, a strong ion pairing propensity leads to a lower osmotic coefficient, hence the minimum in the osmotic coefficients when the solvation free energies of the cation and anion match. This 'law' explains why fluoride salts follow a reverse trend to the other halide anions, i.e., they pair most strongly with smaller cations. This reverse trend is also observed for hydroxide and is reproduced with the model here. This is not surprising given that hydroxide has a very similar solvation free energy and size compared with fluoride ${ }^{20}$ and the key parameters which determine the ion-water interaction in the model are the ion size and the total solvation free energy. However, there is a significant quantitative difference between fluoride and hydroxide. The alkali hydroxide osmotic coefficients are higher than the corresponding alkali fluorides, indicating that hydroxide has a less favourable interactions with the alkali ions than fluoride. Interestingly, the strongest pair formation is for $\mathrm{LiOH}$ where the cation and anion do not actually have matching water affinity, this demonstrates the limitation of the 'law' of matching water affinity. In contrast, the model used here reproduces this effect reasonably well.

Because the solvation free energy and the size are very similar for the fluoride and hydroxide ions, the reason for the difference in their osmotic coefficients is presumably not attributable to a difference in the ion-water interaction. The reason instead, is due to the fact that fluoride has a stronger direct short range interaction with the alkali cations than hydroxide does. This effect is predicted by the COSMO calculations as shown in Figure 8, which compares the sodium hydroxide PMF with the PMF for sodium fluoride. It is clear that the pairing is qualitatively similar but with the contact minimum somewhat higher for the hydroxide case. This demonstrates the importance of including direct high level calculation of ion-ion interactions in the model and shows that this may be more important than including an atomically accurate description of the hydration structure around these ions. This also demonstrates the importance of direct quantum mechanical calculation of the ion-ion interaction, as a simple model would predict a stronger interaction for hydroxide 


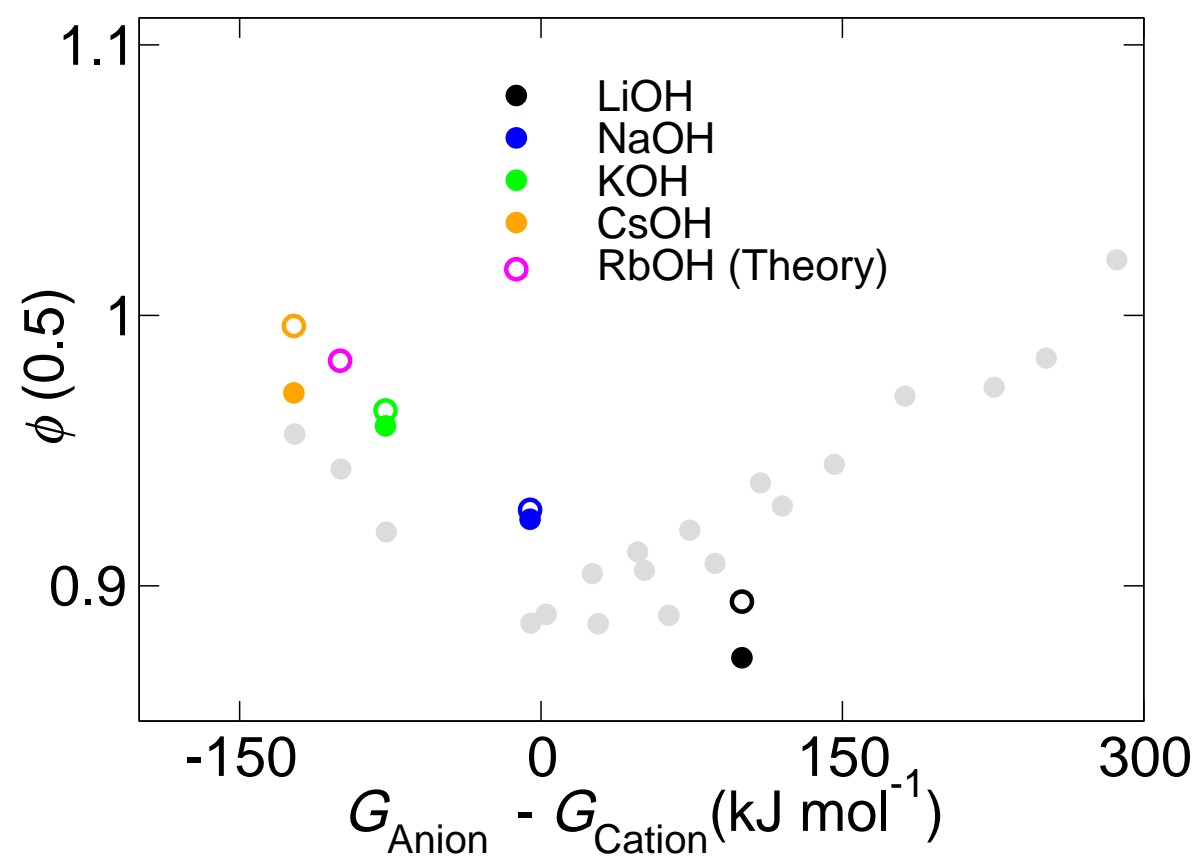

Figure 7: Experimental osmotic coefficients at $0.5 \mathrm{M}$ as a function of difference in solvation free energy between the cation and the anion. Experimental values are filled in circles, theoretical CSM values are open circles. Alkali halides are in grey. 
due to its dipole moment in contrast to the observation here. One possible explanation is the weaker interaction is an entropic effect due to the strong angular dependance of the $\mathrm{Na}-\mathrm{OH}$ interaction compared with $\mathrm{Na}-\mathrm{F}$.

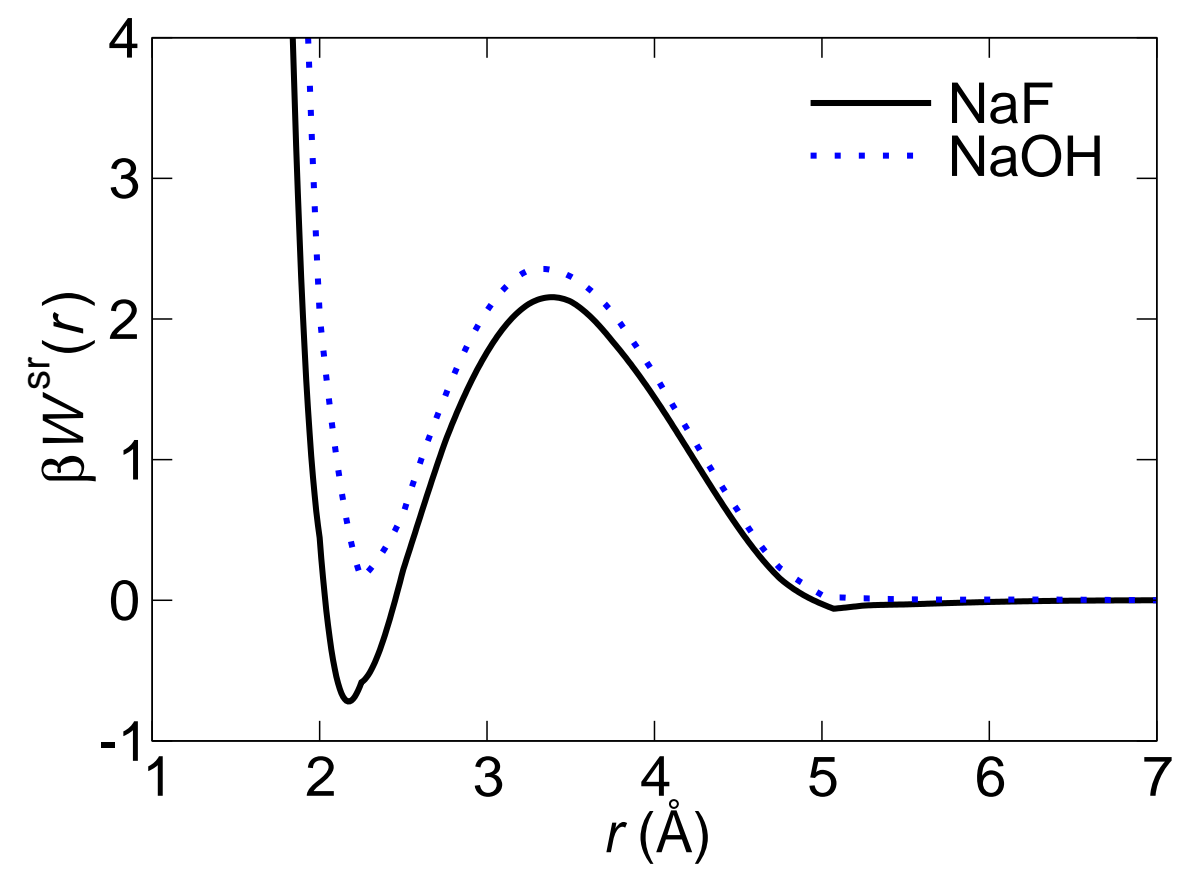

Figure 8: Short range interaction potential of sodium hydroxide compared with sodium fluoride.

There are some contributions this model neglects such as long range charge dipole interactions, which may not be accurately accounted for with the COSMO model. Additionally, the corrections introduced to account for the neglect of charge transfer to the water molecules are crude and could be improved through careful comparison and parameterisation with explicit solvent quantum mechanical calculations. A detailed description of like charge interactions should also be included. It is likely that additional improvements such as including this effect would improve the performance of the theory further.

Nonetheless, the result presented here provides support for the notion that simple approximate models like the continuum solvent approximation can be useful for predicting the properties of electrolyte solutions. The reason for this is that although the model only 
requires two parameters, only one of which was explicitly parameterised to reproduce experimental activity coefficients, it is possible that during the development of this model implicit fitting could have occurred due to the subjective choices which were informed by what gave better experimental agreement. This work is not subject to this problem as exactly the same method and parameters developed and published previously were applied. This means that there is no room for subjective choices to influence the predictions.

The final reason this result is significant is that the hydroxide ion contrasts remarkably with the alkali halide ions. It is a polyatomic, anisotropic and polar molecule. The fact that this model still works for this very different ion is good evidence of the model's transferability. The specific hydrogen bonding structures of water molecules around a hydroxide ion are often emphasised implying that these are very important for determining this ion's thermodynamic properties. The success of this simple approach implies otherwise. This fact also has implications more broadly for our understanding of what it is most important to understand about water. Specifically, it is often assumed that knowing the explicitly resolved atomistic details of the hydrogen bonding structure around solutes is essential to understand their properties. However, this is contradicted by the fact that the osmotic/activity coefficients of the hydroxide ion can be reproduced well using a simple model that ignores essentially all of the details of the explicit water structure.

\section{Conclusion}

We have shown that the osmotic/activity coefficients of the alkali hydroxides ions can be well reproduced using a simple and computationally cheap continuum solvent model without any fitting parameters or modifications to the model. This is significant because of the ubiquitous and important nature these electrolytes in a huge range of important phenomena and applications. The good agreement of this model with experimental results indicates that the model, which was previously developed for the alkali halide ions, is generalisable to 
system different to the original ions it was developed for. It also implies that the details of the specific hydrogen bond networks formed in water around the ions are potentially not as important as other factors such as the direct quantum mechanical interaction between the two ions.

\section{Calculation Details}

\section{Continuum Solvent Model}

The calculation details are very similar to those used in Ref. 27. We used the TURBOMOLE package (v6.4) 28,29 with COSMO ${ }^{30,31}$ implemented. The def2-QZVPP ${ }^{32-35}$ basis sets, and associated ECPs ${ }^{36,37}$ were used for the alkali and hydroxide ions. The calculations were performed both at the Hartree-Fock level using the DSCF program ${ }^{38}$ and at the MP2 level using the RIMP2 program. ${ }^{39-41}$

The cavity sizes for the alkali ions adjusted to match solvation free energies were used as given in Ref. 11 and the hydroxide cavity size was $1 / 79 \AA{ }^{20}$ COSMO's RSOLV parameter was set to $0.84 \AA$. The outlying charge correction was included although the ROUTF parameter had to be reduced to 0.3 , due to numerical error with the default value. The epsilon parameter was set to 116.95 to reproduce the correct 1/78.3 damping of the Coulomb interaction. The ion-specific short-range interaction has a negligible dependence on this parameter.

\section{DFT-MD simualtion details}

The system contains a four $\mathrm{Na}^{+}$four $\mathrm{OH}^{-}$ions and 89 water molecules in a $14.3^{3} \AA^{3}$ supercell. This corresponds to a mole fraction of 0.045 and a Molarity of 2.3 M. NVT (at 300 K) simulation was performed under periodic boundary conditions using CP2K simulation suite (http:www.cp2k.org) with the QuickStep module for the DFT calculations. ${ }^{42}$ The protocol given in Ref., ${ }^{43}$ using a double zeta basis set that has been optimized for the condensed 
phase $^{44}$ in conjunction with GTH pseudopotentials ${ }^{45}$ using a 400 Ry cutoff for the auxiliary plane wave basis is followed. A Nosé-Hoover thermostat was attached to every degree of freedom to ensure equilibration. ${ }^{46}$ The revised Perdew, Burke, and Ernzerhof functional with Grimme dispersion correction was used. (revPBE-D3) ${ }^{47-49}$ Data was accumulated for 190 ps following a 10 ps equilibration.

\section{Osmotic coefficients calculation}

We follow Ref. 50, which utilises the Hyper-netted Chain (HNC) closure of the OrnsteinZernike (OZ) equation to calculate the osmotic coefficients of electrolyte solutions at finite concentration using the infinite dilution PMF. To do this we use the pyOZ program ${ }^{51}$ developed by Luboš Vrbka, an iterative OZ equation solver. Following Ref. 52 and 22, we use a concentration dependent form of the dielectric function of water to account approximately for many-body effects that will become more important as the ion concentration increases:

$$
\epsilon(c)=\frac{78.3}{1+0.19 c}
$$

where $c$ is the concentration of salt in Molarity, and 0.19 is a constant determined by fitting to experimental data for six different salts and averaging. ${ }^{22}$

Experimental densities ${ }^{53-55}$ were used to convert osmotic coefficients to the Macmillan Mayer scheme to enable comparison with the continuum solvent model values. 56,57

\section{Acknowledgements}

XSZ and TTD acknowledge the Australian Research Council (ARC) funding via project number DE200100794, DP200102573 and FL170100101. This research was undertaken with the assistance of resources and services from the National Computational Infrastructure (NCI), which is supported by the Australian Government and with the assistance of re-

sources from QCIF (http://www.qcif.edu.au) and The University of Queensland's Research 
Computing Centre (RCC). Thanks to Christopher Mundy and Gregory Schenter for helpful discussions.

\section{References}

(1) Wang, Y.; Song, Y.; Xia, Y. Electrochemical capacitors: mechanism, materials, systems, characterization and applications. Chem. Soc. Rev. 2016, 45, 5925-5950.

(2) Rajkumar, M.; Hsu, C. T.; Wu, T. H.; Chen, M. G.; Hu, C. C. Advanced materials for aqueous supercapacitors in the asymmetric design. Prog. Nat. Sci. Mater. Int. 2015, $25,527-544$.

(3) Akinwolemiwa, B.; Peng, C.; Chen, G. Z. Redox Electrolytes in Supercapacitors. J. Electrochem. Soc. 2015, 162, A5054-A5059.

(4) Dubouis, N.; Lemaire, P.; Mirvaux, B.; Salager, E.; Deschamps, M.; Grimaud, A. The role of the hydrogen evolution reaction in the solid-electrolyte interphase formation mechanism for ": Water-in-Salt " electrolytes. Energy Environ. Sci. 2018, 11, 34913499.

(5) Keith, D. W.; Holmes, G.; St. Angelo, D.; Heidel, K. A process for capturing $\mathrm{CO}_{j} \operatorname{sub}_{\mathrm{i}} \mathrm{2}_{\mathrm{i}} / \mathrm{sub}_{\mathrm{i}}$ from the atmosphere. Joule 2018, 2, 1573-1594.

(6) Rabinowitz, J. A.; Kanan, M. W. The future of low-temperature carbon dioxide electrolysis depends on solving one basic problem. Nat. Commun. 2020, 11, 5231.

(7) Birdja, Y. Y.; Pérez-gallent, E.; Figueiredo, M. C.; Göttle, A. J.; Calle-vallejo, F.; Koper, M. T. M. Advances and challenges in understanding the electrocatalytic conversion of carbon dioxide to fuels. Nat. Energy 2019, 4, 732-745.

(8) Huang, J. E. et al. CO2 electrolysis to multicarbon products in strong acid. Science (80-. ). 2021, 372, 1074-1078. 
(9) Robinson, R. A.; Stokes, R. H. Electrolyte solutions; Butterworth \& Co.: Devon, 1959.

(10) Duignan, T. T.; Parsons, D. F.; Ninham, B. W. Collins's rule, hofmeister effects and ionic dispersion interactions. Chem. Phys. Lett. 2014, 608, 55-59.

(11) Duignan, T. T.; Parsons, D. F.; Ninham, B. W. A continuum solvent model of ion-ion interactions in water. Phys. Chem. Chem. Phys. 2014, 16, 22014-22027.

(12) Medda, L.; Carucci, C.; Parsons, D. F.; Ninham, B. W.; Monduzzi, M.; Salis, A. Specific Cation Effects on Hemoglobin Aggregation below and at Physiological Salt Concentration. Langmuir 2013, 29, 15350-15358.

(13) Logan, S. R. Effects of ionic strength on the rates of reaction between ions in solution. Trans. Faraday Soc. 1967, 63, 3004-3008.

(14) May, P. M.; Rowland, D. Thermodynamic Modeling of Aqueous Electrolyte Systems: Current Status. J. Chem. Eng. Data 2017, 62, 2481-2495.

(15) Vaque Aura, S.; Roa Pinto, J.-S.; Ferrando, N.; de Hemptinne, J.-C.; ten Kate, A.; Kuitunen, S.; Diamantonis, N.; Gerlach, T.; Heilig, M.; Becker, G.; Brehelin, M. Data Analysis for Electrolyte Systems: A Method Illustrated on Alkali Halides in Water. J. Chem. Eng. Data 2021,

(16) Pitzer, K. S.; Mayorga, G. Thermodynamics of Electrolytes. II. Activity and Osmotic Coefficients for Strong Electrolytes with One or Both Ions Univalent. J. Phys. Chem. 1973, 77, 2300-2308.

(17) Baer, M. D.; Kuo, I.-F. W.; Tobias, D. J.; Mundy, C. J. Toward a Unified Picture of the Water Self-Ions at the Air-Water Interface: A Density Functional Theory Perspective. J. Phys. Chem. B 2014, 118, 8364-8372. 
(18) Hellström, M.; Behler, J. Structure of aqueous NaOH solutions: Insights from neuralnetwork-based molecular dynamics simulations. Phys. Chem. Chem. Phys. 2017, 19, 82-96.

(19) de Oliveira, D. M.; Bredt, A. J.; Miller, T. C.; Corcelli, S. A.; Ben-Amotz, D. Spectroscopic and Structural Characterization of Water-Shared Ion-Pairs in Aqueous Sodium and Lithium Hydroxide. J. Phys. Chem. B 2021,

(20) Duignan, T. T.; Parsons, D. F.; Ninham, B. W. Hydronium and hydroxide at the airwater interface with a continuum solvent model. Chem. Phys. Lett. 2015, 635, 1-12.

(21) Duignan, T. T.; Baer, M. D.; Mundy, C. J. Ions interacting in solution: moving from intrinsic to collective properties. Curr. Opin. Colloid Interface Sci. 2016, 23, 58-65.

(22) Kalcher, I.; Dzubiella, J. Structure-Thermodynamics Relation of Electrolyte Solutions. J. Chem. Phys. 2009, 130, 134507.

(23) Duignan, T.; Schenter, G. K.; Fulton, J.; Huthwelker, T.; Balasubramanian, M.; Galib, M.; Baer, M. D.; Wilhelm, J.; Hutter, J.; Ben, M. D.; Zhao, X. S.; Mundy, C. J. Quantifying the hydration structure of sodium and potassium ions: taking additional steps on Jacob's Ladder. Phys. Chem. Chem. Phys. 2020, 22, 10641-10652.

(24) Duignan, T. T.; Schenter, G.; Mundy, C. J.; Zhao, X. S. A method for accurately predicting solvation structure. J. Chem. Theory Comput. 2020, 16, 5401-5409.

(25) Duignan, T. T.; Kathmann, S. M.; Schenter, G. K.; Mundy, C. J. Toward a FirstPrinciples Framework for Predicting Collective Properties of Electrolytes. Acc. Chem. Res. 2021, doi/10.1021/acs.accounts.1c00107.

(26) Collins, K. D. Why continuum electrostatics theories cannot explain biological structure, polyelectrolytes or ionic strength effects in ion-protein interactions. Biophys. Chem. 2012, 167, 33-49. 
(27) Duignan, T. T.; Parsons, D. F.; Ninham, B. W. Ion interactions with the air-water interface using a continuum solvent model. J. Phys. Chem. B 2014, 118, 8700-8710.

(28) Turbomole V6.4 2012. A Development of University of Karlsruhe and Forschungszentrum Karlsruhe GmbH; available from http://www.turbomole.com.

(29) Ahlrichs, R.; Bär, M.; Häser, M.; Horn, H.; Kölmel, C. Electronic Structure Calculations on Workstation Computers: The Program System Turbomole. Chem. Phys. Lett. 1989, 162, 165-169.

(30) Klamt, A.; Schüürmann, G. COSMO: A New Approach to Dielectric Screening in Solvents with Explicit Expressions for the Screening Energy and its Gradient. J. Chem. Soc. Perkin Trans. 2 1993, 5, 799-805.

(31) Klamt, A. The COSMO and COSMO-RS Solvation Models. Wiley Interdiscip. Rev. Comput. Mol. Sci. 2011, 1, 699-709.

(32) Weigend, F.; Furche, F.; Ahlrichs, R. Gaussian Basis Sets of Quadruple Zeta Valence Quality for Atoms H-Kr. J. Chem. Phys. 2003, 119, 12753-12762.

(33) Weigend, F.; Ahlrichs, R. Balanced Basis Sets of Split Valence, Triple Zeta Valence and Quadruple Zeta Valence Quality for H to Rn: Design and Assessment of Accuracy. Phys. Chem. Chem. Phys. 2005, 7, 3297-3305.

(34) Hättig, C. Optimization of Auxiliary Basis Sets for RI-MP2 and RI-CC2 Calculations: Core-Valence and Quintuple- $\zeta$ Basis Sets for H to Ar and QZVPP Basis Sets for Li to Kr. Phys. Chem. Chem. Phys. 2005, 7, 59-66.

(35) Hellweg, A.; Hättig, C.; Höfener, S.; Klopper, W. Optimized Accurate Auxiliary Basis Sets for RI-MP2 and RI-CC2 Calculations for the Atoms Rb to Rn. Theor. Chem. Acc. 2007, 117, 587-597. 
(36) Leininger, T.; Nicklass, A.; Küchle, W.; Stoll, H.; Dolg, M.; Bergner, A. The Accuracy of the Pseudopotential Approximation: Non-Frozen-Core Effects for Spectroscopic Constants of Alkali Fluorides XF (X = K, Rb, Cs). Chem. Phys. Lett. 1996, 255, 274-280.

(37) Peterson, K. A.; Figgen, D.; Goll, E.; Stoll, H.; Dolg, M. Systematically Convergent Basis Sets with Relativistic Pseudopotentials. II. Small-Core Pseudopotentials and Correlation Consistent Basis Sets for the Post-d Group 16-18 Elements. J. Chem. Phys. 2003, 119, 11113-11123.

(38) Häser, M.; Ahlrichs, R. Improvements on the Direct SCF Method. J. Comput. Chem. 1989, 10, 104-111.

(39) Weigend, F.; Häser, M. RI-MP2: First Derivatives and Global Consistency. Theor. Chem. Acc. 1997, 97, 331-340.

(40) Weigend, F.; Häser, M.; Patzelt, H.; Ahlrichs, R. RI-MP2: Optimized Auxiliary Basis Sets and Demonstration of Efficiency. Chem. Phys. Lett. 1998, 294, 143-152.

(41) Ángyán, J. G. Choosing Between Alternative MP2 Algorithms in the Self-Consistent Reaction Field Theory of Solvent Effects. Chem. Phys. Lett. 1995, 241, 51-56.

(42) VandeVondele, J.; Krack, M.; Mohamed, F.; Parrinello, M.; Chassaing, T.; Hutter, J. Quickstep: Fast and accurate density functional calculations using a mixed gaussian and plane waves approach. Comput. Phys. Commun. 2005, 167, 103-128.

(43) Pluhařová, E.; Baer, M. D.; Mundy, C. J.; Schmidt, B.; Jungwirth, P. Aqueous CationAmide Binding: Free Energies and IR Spectral Signatures by Ab Initio Molecular Dynamics. J. Phys. Chem. Lett. 2014, 5, 2235-2240.

(44) VandeVondele, J.; Hutter, J. Gaussian basis sets for accurate calculations on molecular systems in gas and condensed phases. J. Chem. Phys. 2007, 127, 114105. 
(45) Goedecker, S.; Teter, M.; Hutter, J. Separable dual-space Gaussian pseudopotentials. Phys. Rev. B 1996, 54, 1703-1710.

(46) Martyna, G. J.; Klein, M. L.; Tuckerman, M. Nosé-Hoover chains: the canonical ensemble via continuous dynamics. J. Chem. Phys. 1992, 97, 2635-2643.

(47) Perdew, J. P.; Burke, K.; Ernzerhof, M. Generalized gradient approximation made simple. Phys. Rev. Lett. 1996, 77, 3865-3868.

(48) Zhang, Y.; Yang, W. Comment on Generalized gradient approximation made simple. Phys. Rev. Lett. 1998, 80, 890-890.

(49) Grimme, S.; Antony, J.; Ehrlich, S.; Krieg, H. A consistent and accurate ab initio parametrization of density functional dispersion correction (DFT-D) for the 94 elements H-Pu. J. Chem. Phys. 2010, 132, 154104.

(50) Vrbka, L.; Lund, M.; Kalcher, I.; Dzubiella, J.; Netz, R. R.; Kunz, W. Ion-specific thermodynamics of multicomponent electrolytes: A hybrid HNC/MD approach. J. Chem. Phys. 2009, 131, 154109.

(51) Vrbka, L. http://pyoz.vrbka.net. 2008.

(52) Hess, B.; Holm, C.; van der Vegt, N. Modeling Multibody Effects in Ionic Solutions with a Concentration Dependent Dielectric Permittivity. Phys. Rev. Lett. 2006, 96, 147801.

(53) Herrington, T. M.; Pethybrldge, A. D.; Roffey, M. G. Densities of Aqueous Lithium, Sodium, and Potassium Hydroxides from 25 to $75 \mathrm{C}$ at 1 atm. J. Chem. Eng. Data 1986, 31, 31-34.

(54) Sipos, P. M.; Hefter, G.; May, P. M. Viscosities and densities of highly concentrated aqueous $\mathrm{MOH}$ solutions $(\mathrm{M}+=\mathrm{Na}+, \mathrm{K}+, \mathrm{Li}+, \mathrm{Cs}+,(\mathrm{CH} 3) 4 \mathrm{~N}+)$ at 25.0C. J. Chem. Eng. Data 2000, 45, 613-617. 
(55) Lide, D. R., Ed. CRC Handbook of Chemistry and Physics, Internet Version, eighty sev ed.; Taylor and Francis: Boca Raton, FL, 2007.

(56) Friedman, H. L. Lewis-Randall to McMillan-Mayer Conversion for the Thermodynamic Excess Functions of Solutions. Part II. Excess Energy and Volume. J. Solution Chem. 1972, 1, 413-417.

(57) Simonin, J.-P. Study of Experimental-to-McMillan-Mayer Conversion of Thermodynamic Excess Functions. J. Chem. Soc. Faraday Trans. 1996, 92, 3519-3523. 